\title{
Inhomogeneous Chemical Evolution of r-process Elements in the Galactic Halo
}

\author{
Benjamin Wehmeyer $^{1}$, Marco Pignatari ${ }^{2}$, and Friedrich-Karl Thielemann ${ }^{1}$ \\ ${ }^{1}$ Univ. Basel, Dept. Phys., Klingelbergstr. 82, CH-4056 Basel, Switzerland \\ ${ }^{2}$ E.A. Milne Centre for Astrophysics, Dept. of Physics \& Mathematics, University of Hull, \\ HU6 7RX, United Kingdom \\ E-mail: benjamin.wehmeyer@unibas.ch
}

(Received August 7, 2016)

\begin{abstract}
For the production of r-process elements in our Galaxy, multiple sites have been discussed, among others, core-collapse supernovae and neutron star mergers. We use the observed elemental abundances of europium $(\mathrm{Eu})$ in metal poor stars to reproduce the galactic chemical evolution of r-process elements. Our main findings are that additionally to neutron star mergers, a second, early acting site is necessary. We assume "magnetorotationally driven supernovae" act as this additional and earlier r-process site and conclude that our simulations with an adequate combination of these two sites successfully reproduces the observed r-process elemental abundances in the Galactic halo.
\end{abstract}

KEYWORDS: nuclear reactions, nucleosynthesis, abundances; Galaxy: abundances; Galaxy: evolution.

\section{Introduction}

The cosmic life cycle depends on many ingredients, including the different stellar production sites, their variations as a function of metallicity, and their occurrence frequency during galactic chemical evolution. Gas clouds form and eventually collapse to form stars. These stars will experince different evolutionary stages according to their individual properties (e.g., [1], $[2])$. They end their life either in planetary nebulae, or in violent events like supernovae or hypernovae / gamma-ray bursts (re-ejecting matter to the interstellar medium), leaving behind either a neutron star or a black hole ( [3], and references therein). Eventually, such compact objects might merge, leading to neutron star mergers, neutron star - black hole mergers or black hole mergers.

Although the production sites and the ejected yields, determining the evolution of the nuclear abundances of many lighter elements are known, the origin of the heaviest elements still remains a puzzle. The relative contributions of the proposed sources (e.g., supernovae, neutron star mergers) are still debated (e.g., [4], [5], [6]).

We use our chemical evolution tool "ICE" [7], [8] to examine the influence of some of the main parameters of the cosmic life cycle. With ICE's high resolution (down to a resolution of $\left.(20 p c)^{3}\right)$ runs, we are able to resolve the impact of abundances in supernova remnants on newly born stars. This approach explains the inhomogeneities in the early Galactic evolution stages and might be an explanation for the observed scatter of r-process elements in low metallicity stars [9]. 


\section{The model}

We set up a simulation for a volume of $(2 k p c)^{3}$. In each time step of $10^{6}$ years, the following calculations are performed.

(1) The star formation rate is calculated via a Schmidt-Kennicutt law (power 1,5)

(2) Star forming cells are chosen randomly, but cells with higher gas content are favoured

(3) The mass of a newly born star is chosen randomly via a Salpeter IMF (with an integrated slope of $-1,35)$

(4) The newly born star inherits the chemical abundances of the ISM out of which it is formed

(5) Considering its mass and metallicity, the life time of a star is calculated

(6) Stars which reached the end of their life time will undergo a nucleosynthesis event, depending on their mass. Low and intermediate mass stars will return their mass via a planetary nebula (or, in a double star system eventually as a type Ia supernova), while high mass stars will explode in a core collapse supernova. In the supernova case, the surrounding ISM is moved (by a Sedov blast wave), until $5 \cdot 10^{4}$ solar masses of ISM are swept-up.

(7) There is a probability that stars in a double star system which have experienced a supernova will trigger a neutron star merger event (after spiralling inwards towards the common center of mass for a delaying "coalescence time")

(8) stars in the surrounding ISM (in the affected cells) inherit the event specific yield

\section{Observations}

We use europium (Eu) as a diagnostic for r-pocess nucleosynthesis. More than $90 \%$ of the $\mathrm{Eu}$ in the solar system and the largest fraction in the Universe is made by the r process (e.g., [10]). The abundance of $\mathrm{Eu}$ is also easier to measure in the spectra of stars compared to other r-process elements [11]. Observations used in the figures to compare with our simulations are taken from the SAGA database (e.g., [12], http://saga.sci.hokudai.ac.jp) where the full list of references can be found.

\section{Results}

According to simulations with neutron star mergers as the exclusive site where r-process nucleosynthesis occurs, it is not possible to explain the observed galactic abundances. Compared to observations in the early galaxy, their contribution to the abundances of r-process elements sets in either too late (at too high metallicities, standard merger probability case), or their too frequent occurrence leads to too high abundances at higher metallicities (close to solar, high merger probability case). The influence of the inspiral time scale of the two neutron stars ("coalescence time") only marginally affects the abundance curve. See figure 1 for illustration.

Recent nucleosynthesis calculations succesfully reproduced an r-process in "magnetorotationally driven supernovae" (or "jet-supernovae" / "collapsars" / "magnetars", e.g., [13], [14]). If one in 1000 supernovae explodes as a Jet-SN (and acts as a second source of r-process elements), this contribution sets already in at lower metallicities. A combined environment with both mentioned nucleosynthesis sites reproduces the observed r-process element abundances. This is shown in figure 2 . 


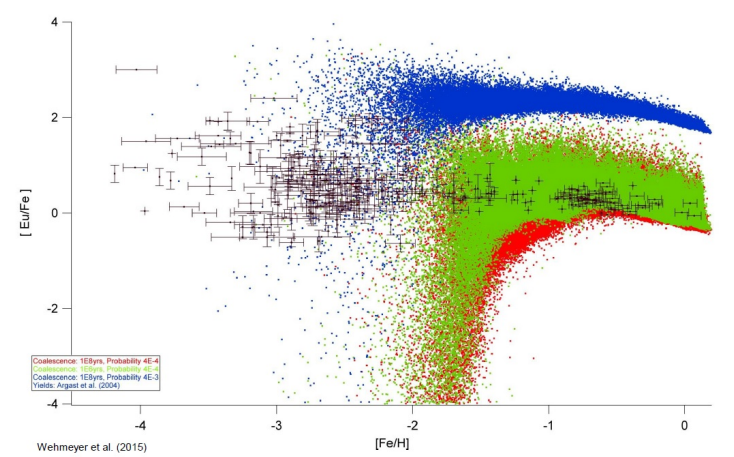

Fig. 1. Effect of probability and coalescence time scale of a simulation with NSM as only rprocess nucleosynthesis site. Grey error bars indicate observations, whereas blue, green, and red dots represent model stars corresponding to environments with different probability or coalescence time scale for NSM events. Figure adopted from $[7]$.

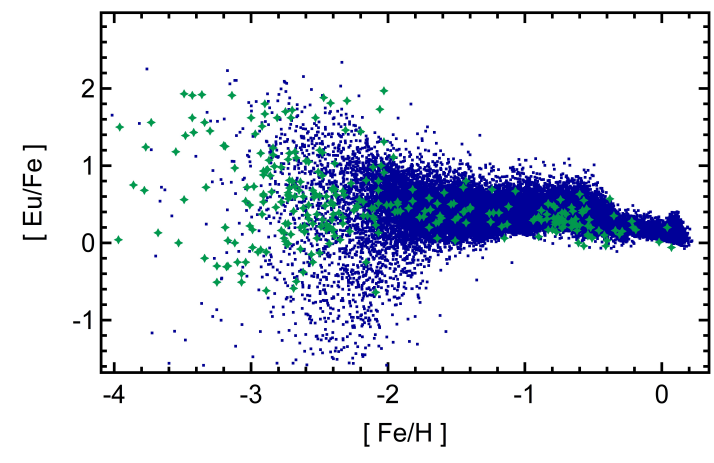

Fig. 2. Abundances of r-process elements in a model with both neutron star mergers and magnetorotanionally driven supernovae as r-process nucleosynthesis sites. Probability of MHD supernovae is one per 1000 regular core-collapse supernovae. Figure adopted from [7].

\section{Acknowledgements}

MP thanks the support from the Swiss National Science Foundation (SNF) and the "Lendület-2014" Programme of the Hungarian Academy of Sciences (Hungary). BW and FKT are supported by the European Research Council (FP7) under ERC Advanced Grant Agreement No. 321263 - FISH, and the Swiss National Science Foundation (SNF). The Basel group is a member in the COST Action New CompStar.

We thank the NIC XIV organizers for a successful conference.

\section{References}

[1] S. E. Woosley, A. Heger, T. A. Weaver, 2002 RvMP 741015

[2] A. I. Karakas, J. C. Lattanzio, 2014 PASA 31 id.e030 62 pp.

[3] N. Langer, 2012 ARA\&A 50107

[4] F. Matteucci, D. Romano, A. Arcones, O. Korobkin, S. Rosswog, 2014 MNRAS 4382177

[5] S. Shen, R. Cooke, E. Ramirez-Ruiz, P. Madau, L. Mayer, J. Guedes, 2015 ApJ 807115

[6] S. Shibagaki, T. Kajino, G. J. Mathews, S. Chiba, S. Nishimura, G. Lorusso, 2016 ApJ 81679

[7] B. Wehmeyer, M. Pignatari, F.-K. Thielemann, 2015 MNRAS 4521970

[8] B. Wehmeyer, M. Pignatari, F.-K. Thielemann, 2016 AIPC 1743 id.040009

[9] I. Roederer, J. Cowan, A. Karakas, K.-L. Kratz, M. Lugaro, J. Simmerer, K. Farouqi, C. Sneden, 2010 ApJ 724975

[10] S. Bisterzo, C. Travaglio, R. Gallino, M. Wiescher, F. Käppeler, 2014 ApJ 787 id.10

[11] C. Sneden, J. J. Cowan, R. Gallino, 2008 ARA\&A 46241

[12] T. Suda, Y. Katsuta, S. Yamada, T. Suwa, C. Ishizuka, Y. Komiya, K. Sorai, M. Aikawa, and M. Y. Fujimoto, 2008 PASJ, 601159

[13] C. Winteler, R. Käppeli, A. Perego, A. Arcones, N. Vasset, N. Nishimura, M. Liebendörfer, F.-K. Thielemann, 2012 ApJ 750 L22

[14] N. Nishimura, T. Takiwaki, F.-K. Thielemann, 2015 ApJ 810 109N 